\title{
L'exercice dans une série de manuels FLE grecs des années 1940-1950 : La méthode Dimitracopoulos
}

The exercise in a series of Greek FFL manuals from the 1940s-1950s: the Dimitracopoulos method

Fryni Kakoyianni-Doa et Monique Monville-Burston

\section{(2) OpenEdition}

Édition électronique

URL : https://journals.openedition.org/dhfles/6877

DOI : $10.4000 /$ dhfles.6877

ISSN : 2221-4038

Éditeur

Société Internationale pour l'Histoire du Français Langue Étrangère ou Seconde

Édition imprimée

Date de publication : 1 décembre 2019

Pagination : 449-471

ISBN : 0992-7654

ISSN : 0992-7654

Référence électronique

Fryni Kakoyianni-Doa et Monique Monville-Burston, «L'exercice dans une série de manuels FLE grecs des années 1940-1950 : La méthode Dimitracopoulos », Documents pour l'histoire du français langue étrangère ou seconde [En ligne], 62-63 | 2019, mis en ligne le 12 avril 2020, consulté le 27 mars 2023. URL : http://journals.openedition.org/dhfles/6877 ; DOl : https://doi.org/10.4000/dhfles.6877

Ce document a été généré automatiquement le 27 mars 2023

Tous droits réservés 


\title{
L'exercice dans une série de
} manuels FLE grecs des années 1940-1950 : La méthode Dimitracopoulos

\author{
The exercise in a series of Greek FFL manuals from the 1940s-1950s: the \\ Dimitracopoulos method
}

Fryni Kakoyianni-Doa et Monique Monville-Burston

\section{Introduction}

1 L'exercice a été/est un élément important dans la pratique pédagogique et l'histoire de l'enseignement des langues. Il est reconnu comme l'un des facteurs primordiaux du développement des compétences linguistiques des apprenants. Les exercices ont en effet toujours fait partie des procédés, supports et activités concrètes utilisés pour promouvoir l'apprentissage et doivent donc être choisis et élaborés avec soin ${ }^{1}$. Comme le dit le Cadre Européen Commun de Référence «les auteurs de manuels, les enseignants et les examinateurs devront prendre des décisions concrètes très précises quant au contenu [...] des exercices, des activités, des tests, etc. [...], les décisions sont, et doivent être, entre les mains des praticiens concernés qui feront appel à leur jugement et à leur créativité » (2001 : 40). Dans le présent article, à visée historique, on pourra se demander comment les exercices qui font partie de manuels de FLE, conçus par un auteur hellénophone et datant des années 1940-1950, manifestent le jugement et la créativité de l'auteur compte tenu des courants méthodologiques ou des décrets officiels de l'époque.

2 Nous examinons ici les exercices inclus dans trois ouvrages de FLE, publiés à Athènes dans les années 1940-1950 environ, et dont l'auteur, Georges Dimitracopoulos², adopte les principes et procédés de la méthodologie directe. Les deux premiers sont des 
manuels (destinés aux niveaux préparatoire et élémentaire des gymnases ${ }^{3}$ ) qui font partie de la série Le français par la lecture et la conversation (dorénavant $F L C$ ). Le troisième est le livre de grammaire Grammaire et Syntaxe (dorénavant GS) qui accompagne la série. La date initiale de parution de ces ouvrages est difficile à déterminer, car nous n'avons pas entre les mains les premières éditions des ouvrages considérés ici. D'après certains indices, nous avons fixé les terminus post quem et terminus ante quem de la publication des diverses éditions des manuels à 1937 et 1958. C'est dans les décrets ministériels de 1929 et 1931 (repris par le décret de 1935) qu'on préconise l'adoption de la méthode directe dans l'enseignement des langues étrangères, méthode dont se réclame Dimitracopoulos. ${ }^{4}$ Il est donc raisonnable de penser que ses ouvrages, qui portent tous en première page l'approbation du ministère de l'Éducation, sont parus après ces dates 5 . D'autre part, le nombre d'éditions est révélateur, dans une certaine mesure: nous possédons par exemple la vingt et unième édition de la méthode $F L C$ du cours préparatoire (dorénavant Prép.) non datée, ainsi que la dixième édition du FLC du cours élémentaire (dorénavant Élém.), datée de 1950. Enfin, Dimitracopoulos a l'habitude, peu ordinaire pour un auteur de manuel, de placer en appendice de ses ouvrages des lettres de personnalités du monde de l'éducation de l'enseignement du FLE qui l'encouragent et le complimentent pour ses travaux : deux de ces lettres, du ministère de l'Éducation français, datent de 1956 et de $1958^{6}$.

3 Le succès de la série $F L C$ et de GS semble avoir été considérable, comme l'indiquent les nombreuses rééditions, et si l'on en croit aussi les extraits de témoignages critiques et d'opinions d'enseignants que Dimitracopoulos a consignés en appendice de ses livres ${ }^{7}$. Ces enseignants ne tarissent pas d'éloges pour FLC et GS qu'ils utilisent, disent-ils, à tous les niveaux du gymnase, qui sont modernes, méthodiques, clairs, simples, bien adaptés à un public hellénophone, avec des textes vivants et bien gradués et des exercices nombreux.

4 Après cette mise en contexte, notre propos est ici d'analyser les exercices contenus dans les manuels FLC (Prép. et Élem.), les deux premiers niveaux de la série, et dans GS. Nous présentons la typologie des exercices présents dans ces ouvrages (lacunaires, d'identification, de transformation, à dominance morphologique, de prononciation, etc.) ainsi que les finalités des différents types d'exercices (mémorisation, application des règles, catégorisation, acquisition des procédures d'analyse du système linguistique, etc.). Nous discutons ces exercices dans le contexte de la méthodologie directe qui oriente la conception des ouvrages de Dimitracopoulos et montrons que, tandis que la GS s'appuie au sens strict et limité sur la méthode directe (aucun recours n'est fait à la langue maternelle), les deux manuels, quant à eux, appliquent plus largement les principes de la méthodologie directe (méthode directe, méthode orale et méthode active).

\section{Les exercices du Français par la lecture et la conversation (Prép. et Élem.)}

Les Prép. et Élém de Dimitracopoulos suivent les principes de la méthodologie directe, celle-ci associant les caractéristiques de trois méthodes principales, directe, active et orale, comme le montre ci-dessous le schéma de Puren $(2012: 81)$ 


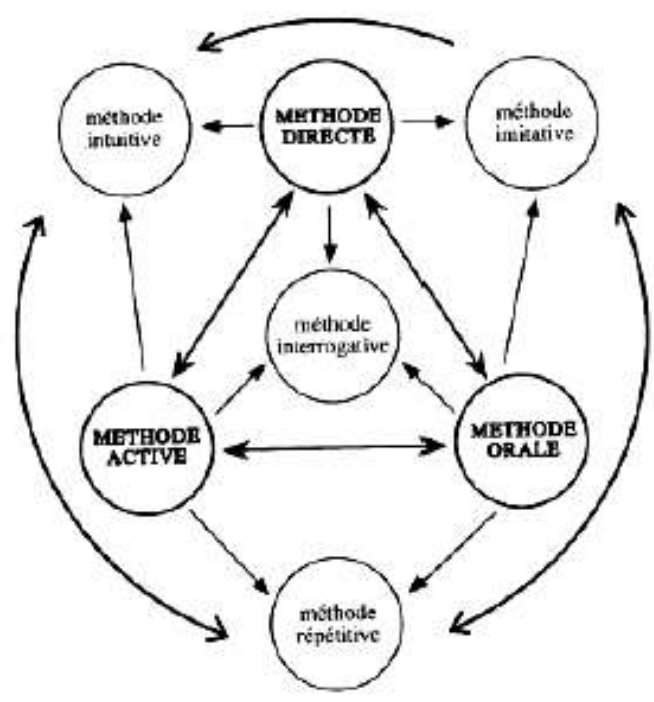

Les grands traits qui la caractérisent peuvent se résumer comme suit. Pour ce qui est de la méthode directe ${ }^{8}:$ utilisation de procédés et de techniques qui cherchent à éviter le passage par l'intermédiaire de la langue maternelle dans l'apprentissage de la langue étrangère -un héritage de la méthode naturelle de François Gouin ${ }^{9}$, la compréhension globale plutôt que celle de mots isolés. Pour la méthode active : utilisation de procédés d'enseignement intuitifs (gestes, aides visuelles), acquisition de la grammaire par des exemples et non des règles, activité de l'apprenant (l'élève apprend à parler en parlant, par un effort personnel pour deviner les sens, en imitant, en répétant, en répondant à des questions qui sollicitent son attention, en réemployant intensivement les formes). Pour la méthode orale : approche audio-orale avec priorité donnée à l'oral sur l'écrit dès le début de l'apprentissage. La méthodologie directe insiste aussi sur une progression linguistique rigoureuse, allant du simple au complexe, du connu à l'inconnu de préférence à un ordre de présentation pragmatique des nouveaux savoirs ${ }^{10}$, et sur l'éveil de l'intérêt de l'élève par un enseignement varié et vivant et par un effort plaisant et non pénible de la part l'élève ${ }^{11}$.

7 Chaque leçon du Français par la lecture et la conversation (Prép. et Élém.) comprend généralement deux parties présentées sur deux pages, trois maximum. La première partie est constituée du texte support de la leçon (conversation, récit, ou, plus rarement, exposé de grammaire) qui permet de réaliser l'intégration didactique de la leçon et son organisation cohérente. Il est accompagné d'un questionnaire qui permet d'exploiter le texte et d'enseigner de nouveaux éléments. La seconde partie comprend les exercices, normalement au nombre de trois. Tous les exercices sont hautement contextualisés, à savoir étroitement liés au texte qui les précède et destinés au réemploi des formes linguistiques enseignées (cf. document 2 ). 
Document 2 : (1950). FLC - Élém. $12^{\mathrm{e}}$ leçon, 28-29

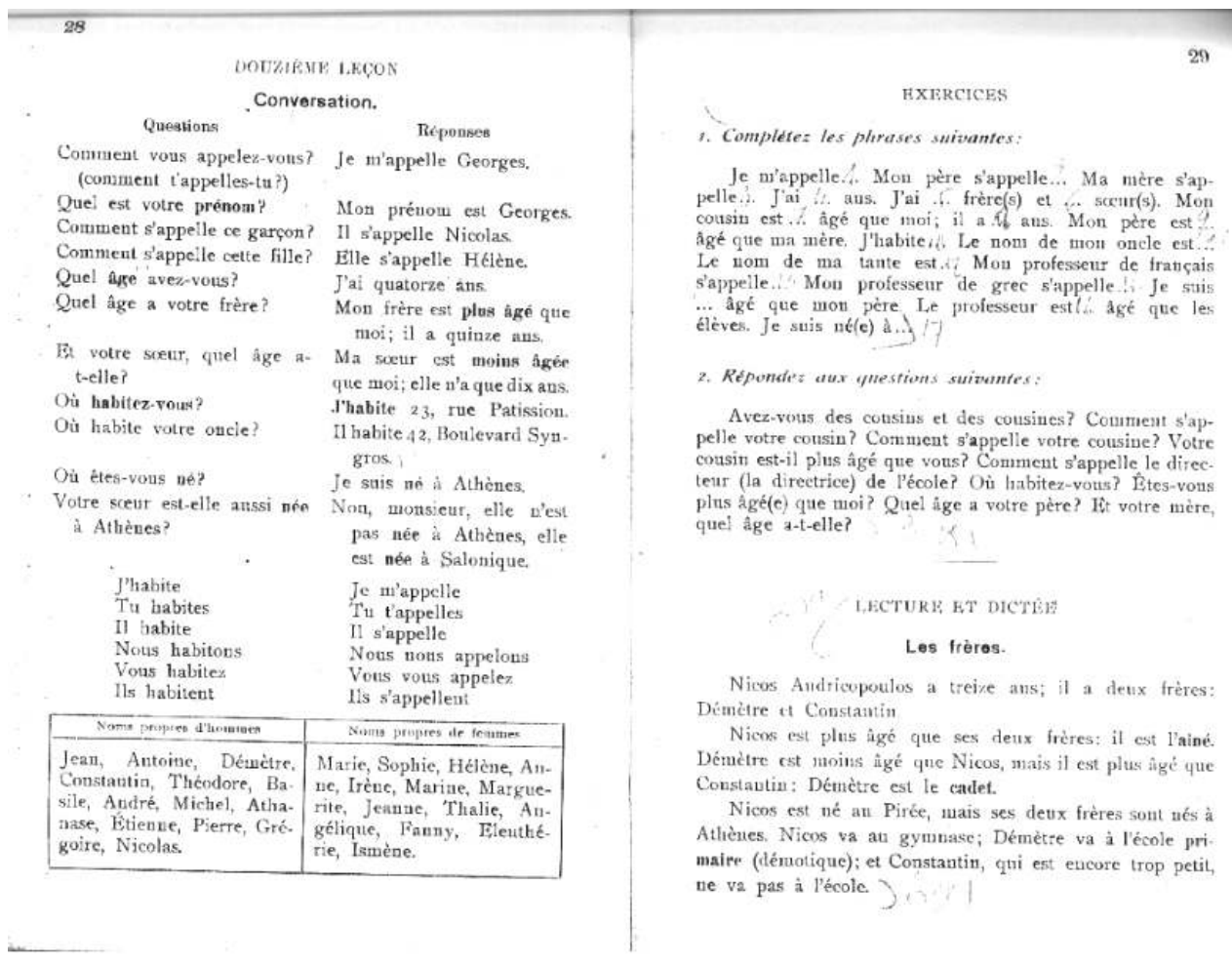

8 Pour les manuels FLC (Prép. et Élém.), Dimitracopoulos précise que les exercices, d'abord faits oralement en classe, puis à l'étude ou à la maison, visent à « développer la faculté expressive des élèves » et les « aident à comprendre et assimiler tout ce qui est dans la leçon ». Ils sont « rédigés à desseins nombreux et variés » $(1950: 5)$. L'auteur insiste sur leur simplicité et sur le gommage délibéré qu'il fait des différences entre le français et le grec. En effet, écrit-il, les livres publiés en France et utilisés pour enseigner le français aux enfants grecs comportent trop d'expressions idiomatiques qui découragent ces jeunes apprenants ${ }^{12}$. Nous verrons que les exercices des deux manuels, adaptés à un public non francophone ${ }^{13}$, reflètent indiscutablement la méthodologie de référence préconisée par l'auteur.

Le nombre d'items par exercice est de six à huit en général, mais peut être variable (particulièrement dans Élém.). Les consignes sont généralement très courtes: "Répondez aux questions qui accompagnent les images; Complétez les phrases suivantes; Lisez les nombres suivants; Prononcez et épelez les mots suivants ", etc. Elles ne répugnent pas à utiliser la terminologie grammaticale : «Le $h$ est-il muet ou aspiré dans homme?; Conjuguez le verbe s'amuser au présent de l'indicatif à la forme négative ", etc. Dans les deux manuels, l'enseignement de la grammaire suit normalement une démarche «inductive implicite» (Besse 2012: 32), mais l'appareillage terminologique (réduit) est présent et de très courtes explications métalinguistiques sont parfois données: "Les adjectifs terminés au masculin en $x$ changent au féminin $x$ en $s e^{14}$; Il y a trois sortes de comparatifs ».

Nous faisons maintenant une typologie des exercices des deux manuels FLC en adoptant certaines des catégories de Vigner 2016 et 2017, pour les deux manuels FLC. On notera qu'il y a 134 exercices pour le niveau élémentaire et seulement 62 pour le niveau préparatoire. 


\section{Exercices lacunaires}

11 Ils sont nombreux, particulièrement dans Élém., et destinés au renforcement aussi bien des connaissances lexicales que grammaticales (Comparer Tableau 1 avec Tableaux 2 et 3) :

Tableau 1 : Exercices lacunaires

\begin{tabular}{|l|l|l|}
\hline Exercices (surtout) écrits & Prép. & Élém. \\
\hline Portant sur la morphologie & 4 & 16 \\
\hline Portant sur le lexique & 5 & 20 \\
\hline Mixtes grammaire-grammaire & 1 & 3 \\
\hline Mixtes grammaire-lexique & 2 & 3 \\
\hline À portée métalinguistique & - & 2 \\
\hline TOTAL & 12 & 44 \\
\hline
\end{tabular}

Lorsque les exercices lacunaires touchent à la morphologie, ils portent surtout sur la forme des différents articles (défini, indéfini, partitif) et sur les formes verbales. Ces exercices à trous sont rarement à choix multiple : on en trouve un seul exemple dans Prép. et deux dans Élém. L'élève doit alors sélectionner des formes qui apparaissent dans la consigne. Par exemple : « Remplacez les tirets par un, une, des, de » ou comme dans le document 3, ci-dessous, « Remplacez les points par plus, aussi ou moins selon le sens ».

Document 3 : (1950). FLC - Élém. 23e leçon, 48

48

\section{EXERCICES}

1. Remplacez les points par plus, aussi ou moins, selon le sens:

Le crayon est... court que la règle. La fenêtre est... large que la porte. Mon bras gauche est... long que mon bras droit. La plume est ... longue que le plumier. Le sac est ... long et ... large que le plumier. La règle est ... longue que la table. Les doigts sont ... courts que les bras. Je suis ... haut(e) que mon père.

Souvent ils sont "mixtes ", c'est-à-dire que la même phrase simple contient plusieurs trous et qu'on demande à l'élève de montrer ses connaissances sur deux points de grammaire à la fois (par exemple forme verbale et pronom personnel), ou sur un point 
de grammaire et un item lexical. Ils sont donc plus complexes en ce qu'ils exigent que l'apprenant ait la capacité de comprendre le contexte et de trouver la partie du discours et le vocabulaire qui conviennent, comme le montre l'exemple suivant ${ }^{15}$ :

(1) Tu ----- ------ à Salonique, tu ----- à Athènes. ------ je derrière ------ tableau ?

La plume ----- elle sur ---- chaise ? Non, monsieur, elle ----- ---- ----- sur -----

chaise, elle ---- sur ---- table. (FLC - Élém.14)

Dans le cas du lexique, l'élément déclencheur de la réponse peut être une image ou un chiffre. On sait l'importance accordée à l'image et aux représentations graphiques dans la méthode directe. Étant donné que toute la leçon se fait en français, certains termes grammaticaux doivent être maîtrisés et font aussi l'objet d'exercices lacunaires, comme le montre l'exemple suivant :

(2) Les lettres $m$ et $n$ sont des ----. La lettre $e$ n'est pas une c----, c'est une ----. Sur le mot boîte, il y a un -----. (FLC - Élém. 17)

\section{Exercices à dominante morphologique}

15 Ils sont nombreux dans Élém. et leur fréquence augmente à mesure que des verbes irréguliers et de nouveaux temps verbaux sont introduits.

Tableau 2 : Exercices à dominante morphologique

\begin{tabular}{|l|l|l|}
\hline Exercices (surtout) écrits & Prép. & Élém. \\
\hline $\begin{array}{l}\text { Mettre des noms/adjectifs au féminin/masculin/pluriel/ singulier ; mettre des } \\
\text { infinitifs au présent/à l'imparfait }\end{array}$ & 5 & 3 \\
\hline Conjuguer des phrases (parfois oralement et par écrit) & 4 & 25 \\
\hline TOTAL & 9 & 28 \\
\hline
\end{tabular}

Badalamenti (2014) appelle ces exercices "exercices de répétition", car la tâche consiste à réitérer une forme donnée dans la consigne. Ils ont été parfois aussi appelés " exercices de maniement du paradigme» (Cristea 1984, citée par Badalamenti). Ils nécessitent en effet l'apprentissage par cœur d'une conjugaison. Un exemple est donné dans le document 4 pour les verbes lire, faire et étudier. 
Document 4 : (1950). FLC - Élém. $21^{\mathrm{e}}$ leçon, 44

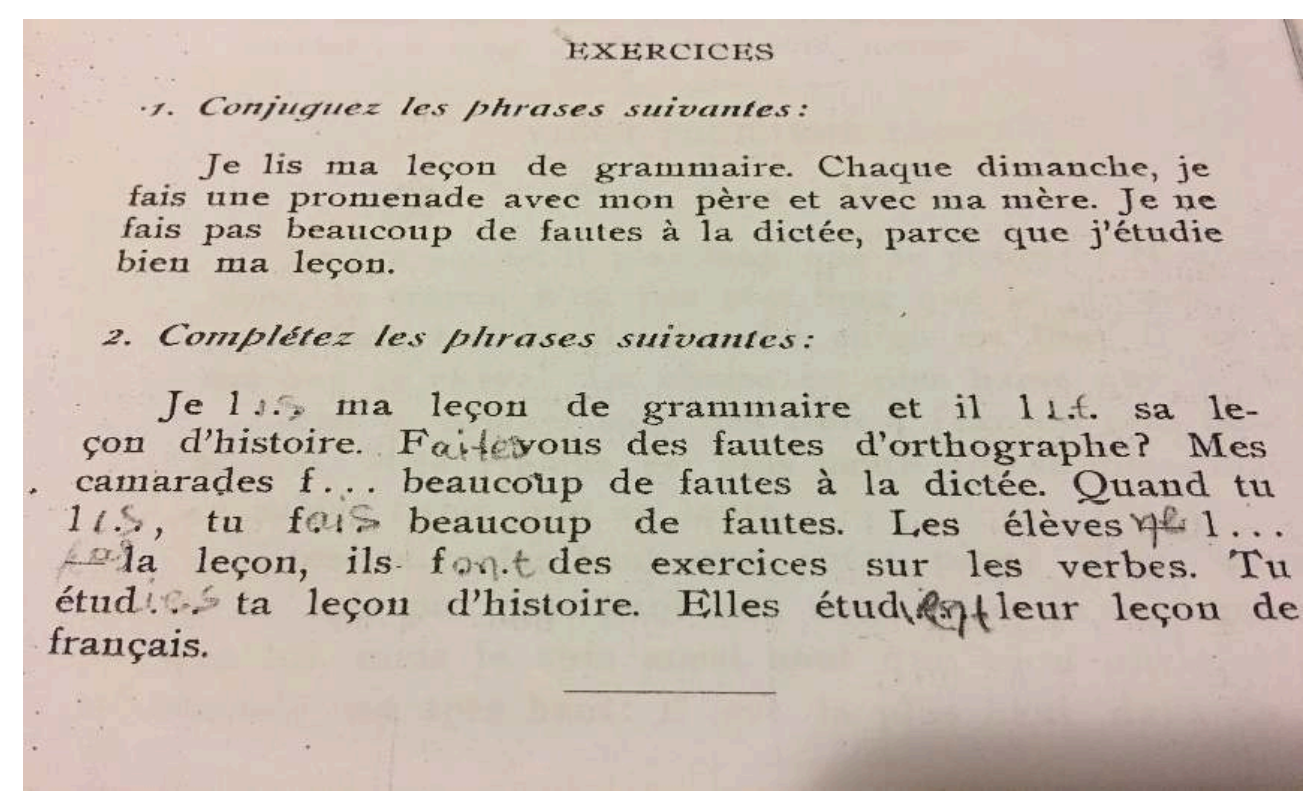

La haute fréquence des exercices liés à la morphologie verbale surtout dans FLC - Élém. est à mettre en rapport avec ce que Dimitracopoulos explique dans sa préface (Élém. 4) : «On remarquera que le verbe occupe une large place dans notre ouvrage : c'est qu'il est la clé de voûte de toute la construction grammaticale». Cette formulation n'est pas sans évoquer la conception sémantico-syntaxique du verbe chez Tesnière (1959): le verbe est hiérarchiquement le plus élevé dans la phrase et organise tous les actants et circonstants ${ }^{16}$.

On observe dans ce type d'exercices sur la morphologie verbale des consignes courantes du type : «Conjuguez les phrases suivantes ». Quelquefois en effet, il ne s'agit pas seulement de conjuguer le verbe, de donner les six formes du paradigme, mais de faire aussi des modifications dans le reste de la phrase. Par exemple, «Conjuguez la phrase : Je lis ma leçon de grammaire ». (Élém. 44).

\section{Exercices de transformation en une phrase nouvelle}

19 Ce type d'exercice est peu fréquent dans Prép. comme on peut le voir dans le tableau cidessous :

Tableau 3 : Exercices de transformation en une phrase nouvelle

\begin{tabular}{|l|l|l|}
\hline Exercices (surtout) écrits & Prép. & Élém. \\
\hline Mettre une phrase à la forme interrogative/négative & 1 & 3 \\
\hline Mettre une phrase au pluriel/au féminin/au singulier & 1 & 14 \\
\hline TOTAL & 2 & 17 \\
\hline
\end{tabular}

Les transformations peuvent être syntaxiques ou morphologiques. Dans le second cas, le changement principal imposé entraîne des changements annexes sur d'autres 
éléments de la phrase : en somme toute la phrase est affectée par le changement de l'une de ses composantes. Par exemple: "Mettez au pluriel: Le cheval est un animal (Élém. 18) ; Cette fille est-elle assise ou debout ?» (Élém. 51).

21 Les catégories d'exercices que nous venons de décrire concernaient particulièrement l'acquisition de l'écrit, sans que l'oral en soit exclu, puisque, comme nous l'avons dit, les élèves peuvent être entraînés à les faire ensemble en classe avant de les reprendre comme devoir à la maison. Nous considérons maintenant des types d'exercices où l'audio-oral prime, et qui, donc, relèvent de la méthode orale dans la méthodologie directe $^{17}$.

\section{Exercices par question/réponse}

Comme le schéma de Puren le montre (doc. 1), la méthode interrogative a une place centrale dans la méthodologie directe : «[...] en classe, les questions orales en langue étrangère sollicitent en permanence l'attention et les réponses orales des élèves directement en langue étrangère" (Puren 2012: 91). Cette forme d'enseignement dialogué où les questions sont " ponctuelles et fermées » a pour but de faire réemployer constamment et systématiquement les formes de la langue étrangère ${ }^{18}$.

Tableau 4 : Exercices par question/réponse

\begin{tabular}{|l|l|l|}
\hline Exercices (surtout) oraux & Prép. & Élém. \\
\hline Questions portant sur le vocabulaire, la vie quotidienne, les connaissances générales & 3 & 35 \\
\hline Questions portant sur la métalangue & 4 & 2 \\
\hline TOTAL & 7 & 37 \\
\hline
\end{tabular}

Les questions posées dans les exercices des deux manuels concernent directement le contenu lexical et grammatical du texte initial de la leçon, mais à la différence des questions contenues dans «Questionnaire » - qui viennent immédiatement après le texte, portent exclusivement sur celui-ci, et ne font pas partie des exercices -, elles peuvent élargir le champ des réponses possibles en interrogeant l'élève, par exemple sur sa vie personnelle: "Combien de minutes marchez-vous pour aller à l'école?» (Élém. 55). Les questions sont parfois d'ordre métalinguistique. Par exemple : «Combien d'accents y a-t-il sur le mot élève? » (Élém. 2).

\section{Production orale de mots isolés/Prononciation}

Ces exercices, où il s'agit de réciter des listes ou de produire un mot unique, ne sont présents que dans Prép., au tout début de l'apprentissage. Ils sont remplacés ensuite par les exercices par question/réponse :

Tableau 5 : Production orale de mots isolés/Prononciation

\begin{tabular}{|l|l|l|}
\hline Exercices (surtout) oraux & Prép. & Élém. \\
\hline
\end{tabular}




\begin{tabular}{|l|l|l|}
\hline Compter à haute voix & 3 & \\
\hline Lire des nombres ou des mots et ensuite les écrire dans le cahier & 2 & \\
\hline Prononcer le mot qui est représenté par une image & 3 & \\
\hline TOTAL & 8 & 0 \\
\hline
\end{tabular}

\section{Exercices oraux/écrits panachés}

Tableau 6 : Exercices panachés

\begin{tabular}{|l|l|l|}
\hline Exercices oraux/écrits panachés & Prép. & Élém. \\
\hline & 24 & 8 \\
\hline
\end{tabular}

Ces sortes d'exercices (fréquents dans Prép.) que nous appelons "panachés» en comparaison avec les précédents, qu'on pourrait dire « uniformes », ne se concentrent pas sur une question, un problème ou un thème uniques, ce qui donne une impression de disparate comme, par exemple, dans l'exercice reproduit ci-dessous (Prép. 83).

1. Conjuguez oralement: J'aime beaucoup mon école.

2. Mettez au masculin : La fille parle à une dame.

3. Écrivez au singulier : Les professeurs sont contents de ces élèves.

4. Copiez les phrases suivantes en les mettant à la forme négative : Vous venez à l'école. Nous aimons ces leçons. Ils travaillent bien.

5. Faites de courtes phrases avec : beaucoup, content, bien, livre, enfant.

Les exercices uniformes correspondent à la définition de Vigner de l'exercice (2017 : 10) : « l'exercice revêt la forme de tâches portant sur une difficulté particulière et une seule et fondées sur un principe de répétition avec une évaluation plus aisée à conduire des réponses obtenues ». Les exercices panachés, au contraire, sont fragmentés : ils rassemblent plusieurs difficultés de nature différente. Ce qui en fait l'unité cependant c'est qu'ils résument en quelque sorte le contenu de la leçon, qu'ils font la synthèse de certains points que le professeur veut qu'on en retienne. Cela tient peut-être aussi au fait que les connaissances des débutants sont limitées (dans Prép.) et ne permettent pas la production d'une grande variété de phrases portant sur une même difficulté ou qu'on souhaite motiver le tout jeune apprenant en lui offrant de la diversité.

Les exercices panachés combinent les types de tâches illustrées ci-dessus (3), mais en incluent d'autres : épellations de mots, orthographe d'homonymes (la vs. là, et vs. est, etc.), lien phonie-graphie : «Trouvez dans le texte 5 mots terminés par une consonne qui ne se prononce pas » (Prép. 93), reconnaissance grammaticale : trouver dans le texte une préposition, une conjonction; souligner les adjectifs possessifs, les adverbes de manière, etc. 


\section{Autres activités orales}

eçons se terminent quelquefois par d'autres activités qui ne sont pas rangées par l'auteur sous la rubrique " exercice». Peut-on cependant les considérer comme des exercices? Ce sont les parties des leçons intitulées (a) «Lecture et dictée » - on en trouve un exemple dans Prép. et quatorze dans Élém. - et (b) «Lecture et récitation » où il s'agit de lire et d'apprendre par cœur une partie du texte initial de la leçon ou un court poème comme dans le document 5 . On en trouve cinq exemples dans Prép. et quatre dans Élém ${ }^{19}$.

Document 5 : (s.d.). FLC - Prep. $13^{e}$ leçon, 53 n peut s'interroger sur les raisons pour lesquelles Dimitracopoulos n'a pas inclus ces activités dans les exercices. Peut-être voit-il dans la récitation une activité de supplément sans lien thématique direct avec la leçon, s'il s'agit de la récitation d'un poème. Cet exercice scolaire depuis longtemps à l'honneur en France, destiné à exercer la mémoire, à améliorer la diction et qui demande que l'apprentissage par cœur soit impeccable, n'est pas facile même pour l'enfant natif et peut être d'autant plus démotivant pour un apprenant de FLE. Ou peut-être l'auteur souhaite-t-il que le professeur utilise cet exercice simplement comme diagnostic, à intervalles réguliers, de la prononciation des élèves. Rien dans les préfaces ne permet de le dire.

Quant à la dictée, il se peut que l'auteur l'écarte aussi des « exercices ", parce que c'est une pure activité de classe, même si elle est préparée chez soi, alors que les exercices sont des devoirs à faire à la maison. Nos exemplaires (annotés) montrent par la mention ochi (non) que certaines dictées ont été écartées du cours par l'utilisateur du manuel, par exemple, dans le document $6^{20}$. 


\section{LECTURE E'T DICTÉE \\ Les frères.}

Nicos Andricopoulos a treize aus; il a deux frères: Démètre et Constantin.

Nicos est plus âgé que ses deux frères: il est l'ainé. Démètre est moins âgé que Nicos, mais il est plus âgé que Constantin: Démètre est le cadet.

Nicos est né au Pirée, mais ses deux frères sont nés à Athènes. Nicos va au gymnase; Démètre va à l'école primaire (démotique); et Constantin, qui est encore trop petit, ne va pas à l'école.

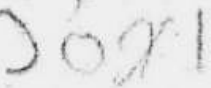

Comme nous venons de le voir dans l'analyse des types d'exercices contenus dans Prép. et dans Élém., l'auteur s'est formellement écarté des principes des méthodologies traditionnelles pour ces niveaux de cours élémentaires. D'abord, on ne trouve aucun exercice de traduction, que l'auteur qualifie dans sa préface $d^{\prime}$ '«inutile et parfois dangereuse" (Élém. 5) ${ }^{21}$. Ensuite, le nombre plus important d'exercices oraux que d'exercices écrits montre que l'accent est mis sur l'acquisition de l'oral et que dans cette approche « les habiletés à lire et à écrire sont développées après l'apprentissage de l'habileté à parler.» (Germain, 1993: 127). De fait, l'écrit n'est pas considéré "comme un système autonome de communication: il s'agit d'une langue orale scripturée, ne faisant que reproduire la langue orale»(ibid.), à savoir une activité subordonnée à l'oral permettant de transcrire ce que l'apprenant sait employer en parlant ( $c f$. questions/réponses, conjugaisons à l'oral, dictée, etc.). Dimitracopoulos note cependant dans la préface à Prép. : « [...] sans nous écarter de la méthode directe, [...] nous faisons marcher de front et vers un même but, par une progression régulière la lecture, l'écriture, la grammaire et l'orthographe de telle sorte que l'une servira désormais à apprendre l'autre. » Le titre des deux manuels en témoigne : Le français par la lecture et la conversation.

\section{Les exercices de Grammaire et syntaxe de la langue française, théorie et exercices à l'usage des élèves grecs}

Grammaire et syntaxe (nous dirions " morphologie et syntaxe ») est le livre de grammaire qui accompagne la série des manuels Le français par la lecture et la conversation. Étant donné le niveau de l'ouvrage, comme nous le verrons dans la suite par les exercices proposés aux élèves, il ne peut être utilisé que par des apprenants d'un niveau intermédiaire ou avancé. Dimitracopoulos note dans la préface, que « partisan de la méthode dite directe ", il a « supprimé les thèmes $»^{22}$. 
Tableau 7 : les exercices dans GS

\begin{tabular}{|l|l|l|l|}
\hline \multicolumn{2}{|l|}{ Type d'exercice } & Fréquence & Total \\
\hline 1. Exercices lacunaires & 23 & 23 \\
\hline & Sans choix multiple & 17 & \\
\cline { 2 - 5 } & Avec choix multiple & 6 & \\
\hline 2. Transformations & 13 & 13 \\
\hline 3. Exercices à dominante morphologique & & 62 \\
\hline & Réitération de la forme & 49 & \\
\cline { 2 - 4 }
\end{tabular}




\begin{tabular}{|c|c|c|}
\hline Accords; choix du temps/mode du verbe & 13 & \\
\hline 4. Corrections de phrases & 13 & 13 \\
\hline 5. Reconnaissance grammaticale : distinctions, repérages, classement & 13 & 13 \\
\hline & & 127 \\
\hline
\end{tabular}

La nature des catégories d'exercices 1,2 et 3 n'est pas différente mutatis mutandis de celle de leurs pendants observés dans Prép. et Élém. Les catégories 4 et 5 méritent quelques remarques.

Sous la catégorie 4, "Corrections de phrase", nous plaçons les exercices libellés "Corrigez, s'il y a lieu les phrases suivantes » et qui peuvent concerner la syntaxe des accords, les constructions verbales ou l'ordre des mots. Par exemple :

- Les tremblements de terre ne sont pas universel; ils sont local.

- Les cahiers qu'il a présenté sont bien tenu et bien écrit.

- Les enfants doivent respecter et obéir à leurs parents.

- L'aviateur est arrivé et parti d'Athènes dans la même journée.

- Je donne à l'élève le livre.

- Télémaque préféra à l'immortalité qu'on lui offrait, sa patrie.

Sous la catégorie 5, «reconnaissance grammaticale» les exercices exigent que l'apprenant ait une bonne maîtrise du métalangage grammatical, sache distinguer les parties du discours, reconnaître les fonctions de celles-ci dans la phrase et en général ait une connaissance solide du système de la langue. Par exemple ${ }^{26}$ :

- Soulignez les pronoms personnels.

- Qualifiez les propositions circonstancielles.

- Soulignez les attributs dans les phrases suivantes et dites s'ils se rapportent au sujet ou au complément.

- Classez les propositions de l'exercice suivant en 3 listes: propositions principales, propositions relatives, propositions complétives. Indiquez la fonction des subordonnées.

$40 \mathrm{Au}$ final, les exercices que nous venons de décrire ont beaucoup en commun avec Exercices sur la grammaire française de M. Grevisse qui datent de la même époque (publiés en 1942) et qui ont été étudiés de près par Badalamenti (2014). Certaines des finalités sont similaires: acquisition de la grammaire par la formulation de règles et l'observation d'exemples; révision des règles apprises et leur application de façon déductive, non pas librement mais sous la contrainte de consignes; mémorisation des formes des paradigmes, avec insistance sur les irrégularités; analyse de la phrase complexe de type logique, et non pas pragmatique; reconnaissance et repérage d'éléments linguistiques selon leurs traits morphosyntaxiques et/ou sémantiques; automatisation des compétences métalinguistiques.

\section{Conclusion}

41 L'examen des exercices de GS et de $F L C$ a mis en évidence que bien qu'appartenant au même cours et rédigés par le même auteur, ils ont été élaborés dans un esprit différent, 
adaptés à des niveaux de compétence différents et destinés à l'acquisition de compétences différentes et que donc ils se distinguent partiellement dans leur nature. Les exercices de GS s'inscrivent dans la ligne de la méthode directe et de la méthodologie de la grammaire traditionnelle: ils sont souvent longs, visent à la maitrise du système de la langue, à l'application de règles, et à l'acquisition d'un niveau assez avancé de compétences métalinguistiques. Les exercices de $F L C$, destinés aux débuts de l'apprentissage sont courts, hétéroclites, associant la pratique de la prononciation, de l'écoute, de la grammaire, du vocabulaire, de l'orthographe et de la métalangue, et suivent fidèlement les principes des composantes de la méthodologie directe (méthodes directe, active et orale).

Si on les compare aux exercices de la méthode traditionnelle qui se limitent à des traductions (thème et version), les exercices de GS et de FLC sont variés et se veulent attrayants pour ne point générer l'ennui. Comme l'a montré Puren (2012 : 78-79), la méthodologie directe est la première à faire appel à des principes psychopédagogiques : en particulier elle tient compte des capacités, des besoins et des intérêts des élèves, elle cherche à susciter leur motivation (une préoccupation constante de Dimitracopoulos signalée dans ses préfaces) en prodiguant un enseignement pratique. Ce sont peut-être les raisons de la présence continue des ouvrages de Dimitracopoulos sur le marché. Leurs nombreuses rééditions sont la preuve de la permanence de leur intérêt pour les enseignants et de l'implantation solide de la méthodologie directe en Grèce immédiatement après la seconde guerre mondiale.

\section{Sources primaires et archives}

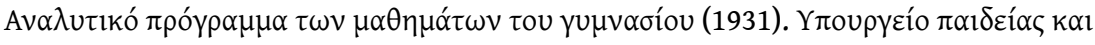
$\Theta \rho \eta \sigma \kappa \varepsilon u \mu \alpha ́ \tau \omega \nu$ [Programme scolaire des cours du gymnase. Ministère de l'Éducation et de Culture religieuse]

Circulaire relative à l'enseignement des langues vivantes (1901, 15 novembre) Signée G. Leygues. B.A.M.I.P., t. LXX, 895-897 (Citée par Puren 2012 : 300).

DIMITRACOPOULOS, Georges D. (entre 1941 et 1945 ?). Grammaire et syntaxe de la langue française, théorie et exercices. Athènes : 29 , rue Tharypou. ( $5^{\mathrm{e}}$ édition).

DimitRACOPOULOS, Georges D. (1950). Le français par la lecture et la conversation - Cours élémentaire. À l'usage des élèves grecs qui n'ont pas été habitués à parler le français dès leur enfance. Athènes : Éditions Georges. (10édition).

DIMITRACOPOULOS, Georges D. (sans date : 1958 ?). Le français par la lecture et la conversation Cours préparatoire. À l'usage des élèves grecs et étrangers qui n'ont pas été habitués à parler le français dès leur enfance. Athènes : Éditions Georges. (21e édition). 


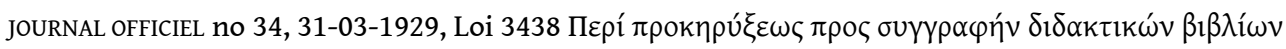

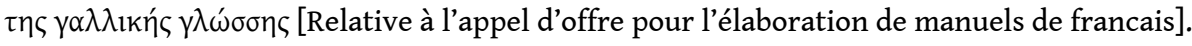

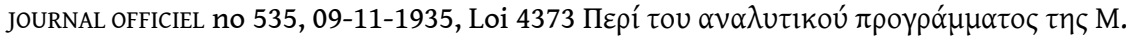

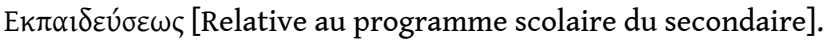

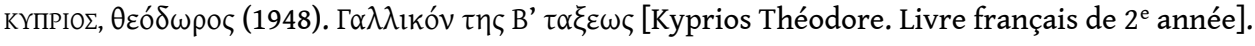

Athènes : Estia.

\section{Sources secondaires}

BADALAMENTI, Rosa Leandra (2014). « Les exercices de grammaire de Grevisse. Analyse des Exercices sur la grammaire française de M. Grevisse, 1942 ». Documents pour l'histoire du français langue étrangère ou seconde 52, 165-180.

BESSE, Henri ([1985] 2012). Méthodes et pratiques des manuels de langues. Paris : Hatier-Credif. BESSE, Henri \& PORQUIER, Rémy (1984). Grammaires et didactique des langues. Paris : Hatier-Credif. CONSEIL DE L'EUROPE (2001). Cadre européen commun de référence pour les langues : apprendre, enseigner, évaluer. Paris : Didier.

CUQ, Jean-Pierre \& GRUCA, Isabelle (2012). Cours de didactique du français langue étrangère et seconde. Grenoble : Presses Universitaires de Grenoble.

DEFAYS, Jean-Marc (2003). Le français langue étrangère et seconde - Enseignement et apprentissage. Sprimont : Mardaga.

EFTHYMIOU, Loukia \& MANITAKIS, Nicolas (2017). « 'La méthode orale' : transferts pédagogiques et pratiques innovantes dans l'enseignement et la didactique du français en Grèce ». Documents pour l'histoire du français langue étrangère ou seconde 58-59, 121-137.

GERMAIN, Claude (1993). Évolution de l'enseignement des langues : 5000 ans d'histoire. Paris : Clé International.

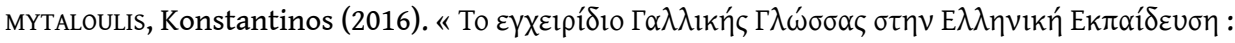

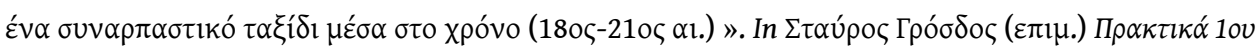

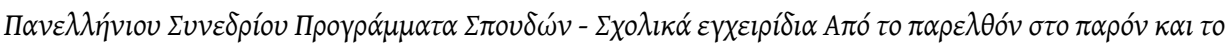

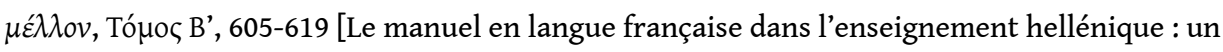
passionnant voyage dans le temps (XVIII $-\mathrm{XXI}^{\mathrm{e}}$ siècles). In Stavros Grosdos (dir.). Actes du Colloque Programme d'Études - Manuels scolaires du passé au présent et au futur, Vol B', 605-619]. Athènes : Musée de la vie scolaire et de l'éducation d'EKEDISY.

PROVATA, Despina \& VIHOU, Marina (2018). « La dimension culturelle dans les manuels illustrés pour enfants : le cas de La collection enfantine de Théodore Kyprios ». Documents pour l'histoire du français langue étrangère ou seconde 60-61, 323-343.

PUREN, Christian ([ $\left.{ }^{1} 1988\right] 2012$ éd. numérisée). Histoire des méthodologies de l'enseignement des langues. (c) Christian PUREN.

TAYLOR, Wilson L. (1953). “Cloze procedure : a new tool for measuring readability”. Journalism Quarterly 30, 415-433.

TESNIÈRE, Lucien (1959). Éléments de linguistique structurale. Paris : Klincksieck. En ligne : [https:// archive.org/details/LucienTesniereElementsDeSyntaxeStructurale]. 
VIGNER, Gérard (2016). « L'exercice ». Carnets Deuxième série - 8. Revue électronique d'Études

françaises. A.P.E.F. En ligne : [http://journals.openedition.org/carnets/1850].

VIGNER, Gérard (2017). Systématisation et maîtrise de la langue : l'exercice en FLE. Paris : Hachette.

\section{NOTES}

1. Cela s'applique à l'époque qui nous intéresse ici, en Grèce. Les consignes du ministère de l'Éducation, destinées aux auteurs de manuels/grammaires, imposent que leurs ouvrages comportent des exercices (cf. par exemple Journal officiel 1931 et 1935).

2. On sait peu de l'auteur si ce n'est qu'il était professeur de français et directeur d'une maison d'édition. Si on se fonde sur les extraits de critiques et d'opinions concernant ses ouvrages, qui sont reproduits par l'auteur à la fin de ceux-ci et qui sont tirés de lettres de collègues, de professeurs d'université grecs et étrangers, d'écrivains et de personnalités du monde de l'enseignement, on devine que Dimitracopoulos était un acteur engagé dans la promotion des études françaises en Grèce.

3. C'est-à-dire des écoles secondaires en Grèce.

4. Certains principes de la méthodologie directe, en particulier l'utilisation dans l'enseignement de ressources auxiliaires visuelles (epoptikí didaskalía) ont été adoptés dès le début de $\mathrm{XX}^{\mathrm{e}}$ siècle ( $c f$. Provata \& Vihou 2018). Quant aux décrets précités, ils utilisent, pour désigner l'enseignement recommandé, l'expression eks amésou ke epoptikí didaskalía où les adjectifs ámesos ( direct») et epoptikós ("visuel ») se réfèrent respectivement à la méthode directe et à la méthode par l'aspect. Le décret de 1935 donne une liste d'exercices relevant de la méthode directe pour les premiers niveaux. Elle inclut des exercices de phonétique (mots et phrases), des exercices de lecture (phrases et descriptions simples au sujet de la vie scolaire), la mémorisation et la récitation de poèmes, des exercices de réécriture (par recopiage à la maison), des dictées, des questions, des réponses à partir de textes enseignés, pour assimiler le lexique, la morphologie et la syntaxe. Il est à noter que des exercices occasionnels de traduction de grec au français sont mentionnés, qui ne ressortissent pas de la méthode directe. Les exercices de la série d'ouvrages de Dimitracopoulos n'en comportent pas.

5. «Ouvrage approuvé par le ministère de l'Instruction publique/Ouvrage approuvé par le Ministère de l'Éducation nationale. » (Prép., Élém., GS)

6. Elles sont signées par les ministres de l'Éducation nationale René Billières et Jean Berthoin (voir Élém.). On peut aussi affirmer que GS était déjà publié dans les années 1941-1945, car une lettre de félicitations est adressée à l'auteur pour son travail, signée par Roger Milliex, Professeur et Secrétaire-Général de l'Institut Français d'Athènes, fonctions qu'il a occupées entre ces deux dates.

7. Selon Mytaloulis (2016), à partir de 1912, la majorité des manuels de FLE utilisés dans l'enseignement public étaient ceux de Théodore Kiprios. Ils ont eu jusqu'à 15 éditions. Mytaloulis signale que par la suite la série FLC a connu un succès similaire, certains des ouvrages ayant atteint la $17^{\mathrm{e}}$ édition.

8. La méthode directe succède à la méthode naturelle en France à la fin du XIX siècle dans l'enseignement des langues. En 1901 la Circulaire relative à l'enseignement des langues vivantes impose l'utilisation de cette méthode dans l'ensemble de la nation (cf. Puren $2012: 64$ ).

9. C'est une approche assimilatrice que l'on retrouve de nos jours dans les programmes d'immersion : l'apprenant est plongé immédiatement dans la langue cible.

10. On notera que la première page de Prép. indique après le titre: "Méthode directe et progressive ». 
11. Nous renvoyons par exemple à Puren 2012, Germain 1993, Defays 2003, Besse 2012, Cuq \& Gruca 2012, pour des discussions élaborées de cette méthodologie.

12. L'auteur cite en exemple l'expression il s'en est allé, qui serait rendue en grec par un seul mot. On peut imaginer que les livres français pour enfants auxquels il fait allusion - Les premiers pas, Lectures enfantines, Premières lectures (Élém. Préface: 4-5) - sont probablement des manuels comme : Rocherolle, Éd. (1862). Les premières lectures enfantines. Paris : Armand Colin ou Bouillot, V. (1910-1920). Lectures enfantines - Lecture écriture vocabulaire. Paris : Hachette.

13. Dans sa préface à Élém. (1950: 5), Dimitracopoulos indique que son ouvrage «convient également à tous les élèves étrangers qui ne sont pas habitués à parler le français dès leur enfance ».

14. Dans l'original, les terminaisons « $\mathrm{x}$ » et « se » ne sont pas en italiques. Nous avons fait cette modification pour améliorer la lisibilité.

15. Ils se rapprochent des tests de closure mis au point par Taylor (1953), utilisés comme instrument de mesure de la compétence à lire un texte. Ces tests sont souvent utilisés dans l'enseignement en immersion, qui a bien des traits communs avec la méthodologie directe. Dans les exercices de $F L C$, cependant, les lacunes ne viennent pas à intervalles réguliers comme dans les tests de closure classiques.

16. Il est intéressant de noter que la parution du Cours élémentaire de syntaxe structurale de Lucien Tesnière date de 1938 et que ses autres publications couvrent les années 40 et 50 . Mais sans plus de preuve et de connaissances sur la réception des œuvres de Tesnière, la coïncidence entre la conception du rôle du verbe chez le didacticien/professeur et le linguiste reste aléatoire.

17. Sur la méthode orale d'enseignement du français en Grèce, immédiatement avant et après la seconde guerre mondiale voir Efthymiou \& Manitakis (2017). C'est à l'Institut français d'Athènes (et seulement dans cet établissement) que cette méthode a été mise en œuvre de façon systématique et à l'état pur avec des conditions d'apprentissage semblables à celles de la langue maternelle, sans manuel pendant deux ou trois ans, avec progression du concret à l'abstrait dans l'acquisition du vocabulaire, utilisation du jeu, de l'image et de la chanson, etc.

18. Cette procédure rigide et directive (question du professeur > réponse de l'élève) a été critiquée comme une faiblesse de la méthode directe (cf. Puren 2012).

19. Il est à noter que les consignes du ministère de l'Éducation qui concernent l'enseignement du français (1935) recommandent la mémorisation et la récitation de poèmes ( $\alpha \pi \alpha \gamma \gamma \varepsilon \lambda i ́ \alpha$ a $\alpha$ ó

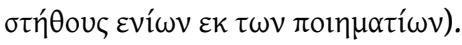

20. On notera aussi que d'après l'instruction de 1902 en France la progression vers la rédaction

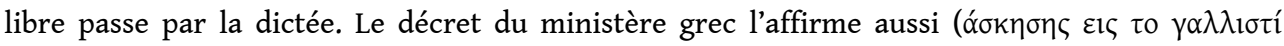

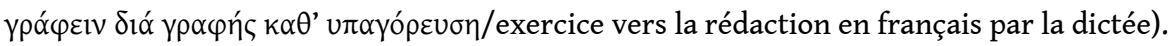

21. Il l'admet cependant pour « les leçons de choses qui désignent des plantes et des animaux ».

22. Le manuel de français de Théodore Kyprios par exemple, contient des phrases de traduction (cf. édition de $1948: 15$ ).

23. Dans Élém. (préface, 4) on lit: «Ce nouvel ouvrage conçu et rédigé dans le même esprit de simplicité, d'ordre et de clarté que notre «Grammaire et Syntaxe de la Langue Française » peut en être considéré comme le complément nécessaire pour l'enseignement du français ».

24. Noter la terminologie utilisée par l'auteur, qui en cela ne s'est pas encore affranchi du vocabulaire grammatical de la méthodologie traditionnelle.

25. Roger Milliex, professeur et secrétaire général de l'Institut d'Athènes dont Dimitracopoulos reproduit la lettre pleine de louanges qui commente son livre, écrit : «Ainsi donc les élèves qui pratiqueront vos ouvrages, sous la conduite d'un maître qui saura s'interdire à lui-même et leur interdire le procédé paresseux et inefficace de la traduction, ne seront pas dépaysés en arrivant chez nous. » Cette condamnation sans appel de la traduction dans l'enseignement montre aussi l'accord entre les vues de Dimitracopoulos et celles de l'établissement le plus en pointe de l'enseignement du français à l'époque en Grèce. 


\section{RÉSUMÉS}

Le présent article examine les exercices inclus dans trois ouvrages de français langue étrangère (FLE), publiés à Athènes dans les années 1940-1950 environ, et dont l'auteur, Georges Dimitracopoulos, adopte les principes et procédés de la méthodologie directe. Les deux premiers sont des manuels (destinés aux niveaux préparatoire et élémentaire des 'gymnases') qui font partie de la série Le français par la lecture et la conversation [FLC]. Le troisième est le livre de grammaire Grammaire et Syntaxe [GS] qui accompagne la série. Les exercices de ces ouvrages étant d'une grande diversité, il en est fait une typologie afin de les classifier et de les définir. Les différents types d'exercices et leur finalité didactique sont examinés à la lumière des trois caractéristiques majeures de la méthodologie directe (méthodes directe, orale et active). On montre que les exercices de GS, quoique relevant de la méthode directe, restent traditionnels, tandis que les exercices des deux manuels de FLC sont d'excellents exemples de l'application des caractéristiques de la méthodologie directe dans son ensemble.

This article examines the exercises included in three French as a foreign language books, published in Athens in the years 1940-1950 approximatively, whose author, Georges Dimitracopoulos, adopts the "direct methodology" principles and procedures. The first two are textbooks (intended for the preparatory and elementary levels "gymnasiums") which are part of the series Le français par la lecture et la conversation [FLC, "French by Reading and Conversation"]. The third is the grammar book Grammaire et Syntaxe [GS, "Grammar and syntax"] which accompanies the series. The exercises in this books being of a great diversity, a typology is established in order to classify and define them. The various types of exercises and their pedagogic purposes are examined in the light of the three main characteristics of the direct methodology ("direct, oral and active methods"). It is shown that the GS exercises, while applying the direct method, remain traditional, while the exercises in both FLC manuals are excellent examples of the application of the characteristics of the direct methodology as a whole.

\section{INDEX}

Keywords : language learning exercises, French as a foreign language textbooks, Greek learners, direct (natural) methodology, direct method, oral method, active method

Mots-clés : exercices de langue, manuels de FLE, élèves grecs, méthodologie, méthodologie directe, méthode directe, méthode orale, méthode active

\section{AUTEURS}

\section{FRYNI KAKOYIANNI-DOA}

Université de Chypre

frynidoa@ucy.ac.cy 


\section{MONIQUE MONVILLE-BURSTON}

Université technologique de Chypre

monique.burston@cut.ac.cy 